\title{
Necropsies in African children: consent dilemmas for parents and guardians
}

K Lishimpi, C Chintu, S Lucas, V Mudenda, J Kaluwaji, A Story, D Maswahu, G Bhat, A J Nunn, A Zumla

Background-Necropsy examination pro-
vides a good index of the accuracy of clinivides a good index of the accuracy of clinical diagnosis and the quality of treatment, but its use in sub-Saharan Africa is limited.

Aims-To identify the main reasons for parents'/guardians' refusal of consent for necropsy and to explore the issues affecting their decision.

Methods-A sequential necropsy study of Zambian children between 2 months and 15 years dying of respiratory disease. When the parent/guardian refused permission for necropsy, the main reason given was recorded, after encouragement to express their specific concerns in their own words.

Results-Parents/guardians of 891 of 1181 children $(75.4 \%)$ refused to give permission, and $290(24.6 \%)$ consented. Of those who refused, $43 \%$ did so on the grounds that it would be a "waste of time," as the diagnosis should have been made in life and the findings would now be of no benefit to them. More than one quarter of those who refused did so because a death certificate had already been issued and de and could not be delayed. Trabade the mutilation of dead bodies were cited by $77(8.6 \%)$. Other reasons included the child not being their own or that they must seek permission from other family members who were not available (6\%). Religious beliefs were not a major cause of refusal.

Conclusions-It is possible to achieve a rate of necropsy consent sufficient to undertake valuable clinical pathology studies on children in sub-Saharan Africa. The wide range of reasons cited for refusal points to the diverse and complex interaction of social and cultural factors affecting attitudes to necropsy examination. Medical staff need training and support to improve the uptake of clinical pathology services.

(Arch Dis Child 2001;84:463-467)

Keywords: children; necropsy; consent; Africa

Professor A Zumla, Centre for Infectious Diseases and International Health, UCL Windeyer Institute of Medical Sciences Room G41, 46 Cleveland Street, London W1P 4JF, UK email: a.zumla@ucl.ac.uk

Accepted 7 March 2001
Necropsy examination provides a good index of the quality of patient care, both in terms of ity of treatment given. In Western countries, the accuracy of clinical diagnosis and the qual- despite the availability of sophisticated diagnostic techniques, there are observed discrepancies between clinical and necropsy diagnoses. In a hospital based study of 1188 necropsies done between 1980 and 1984 at the Cantonal Hospital, Lucerne-where the necropsy rate was $52 \%$ for hospital inpatient deaths-90\% confirmed the clinical diagnosis, $75 \%$ found previously unknown and clinically important findings, and major discrepancies were found in $10 \%$ of cases. ${ }^{1}$ In a further $10 \%$ of cases the necropsy findings would have been of potential therapeutic relevance if they had been detected before death. Such discordance in Western teaching hospitals means that mortality statistics which are unsupported by necropsy findings must be viewed with caution. $^{2}$

There has been a progressive decline in necropsy rates throughout the world. ${ }^{3-7}$ The reasons for this are many, varied, and complex. Clinicians have tended to undervalue the clinical importance of necropsy and, when faced with increasingly litigious relatives, have become wary of requesting an examination that may reveal discrepancies between diagnosis and management. The cost implications of an effective clinical pathology service are significant. There is a global shortage of trained pathologists and insufficient capacity to cover biopsy as well as necropsy workloads, especially in areas facing extreme resource constraints. Additionally, professional and lay perceptions and attitudes towards necropsy have remained unchallenged, and pathologists themselves have not appeared proactive in countering the insidious decline in necropsy rates.

In most African countries, the health services are overwhelmed with the increasing mortality from the devastating effects of HIV. There are overlapping clinical presentations of opportunistic infections and HIV associated tumours, and with the gradual deterioration of health and diagnostic services clinicians are increasingly faced with diagnostic dilemmas. Clinical practice is currently based on probabilities rather than on an agent specific diagnosis. The only way to determine causes of death and develop proper diagnostic algorithms is to conduct necropsy examinations and correlate the findings with the clinical picture. Several previous necropsy studies have generated such data. ${ }^{89}$

In Zambia, as in most countries, necropsy on individuals cannot be performed without first obtaining written permission from the deceased's relatives. Nearly all families have lost a 
close relative from HIV/AIDS and suffer additional distress when a necropsy is requested. This distress is intensified when the request for necropsy examination involves children. Furthermore, the scarcity of trained African pathologists enforces an unacceptable delay before burial, potentially prolonging acute grief for those bereaved, and complicating the funeral arrangements and mourning. Not surprisingly, the majority of families refuse requests for necropsy examination. Verbal necropsies have been suggested as an alternative method for ascertaining causes of childhood mortality in some African countries. ${ }^{10}$ This approach may provide useful information about causes of death outside hospital ${ }^{11}$ but has major limitations for the evaluation of exact causes of death and for health impact evaluations. ${ }^{12} 13$

Necropsy studies from Africa have been scarce, and examination rates in hospitals have historically been very low. Today, necropsy examinations in African hospitals are mostly restricted to specific forensic cases ${ }^{14}$ or to research projects seeking answers to specific questions. ${ }^{9}$ Childhood mortality rates have increased in sub-Saharan Africa in recent years, while the number of necropsy examinations undertaken has remained static or declined. In Zambia, necropsy rates have historically been low- $17 \%$ for all deaths and $10 \%$ for childhood deaths. ${ }^{15}$ As part of a large necropsy study of Zambian children dying of respiratory diseases at the University Teaching Hospital (UTH), Lusaka, Zambia, we took the opportunity to examine the reasons for necropsy refusal by parents and guardians.

\section{Methods}

SETTING

Between 15 September 1997 and 15 June 2000, a sequential necropsy study of Zambian children dying of respiratory disease was carried out in the department of paediatrics and child health, UTH, Lusaka. The university teaching hospital is the only government hospital in Lusaka providing inpatient care for children. Children with illness serious enough to warrant admission are referred from all peripheral clinics serving the city population. All seriously ill Lusaka children warranting hospital admission are seen in the department of paediatrics and child health at UTH. The study covered children from a wide population base (just over 400000 children in Lusaka).

\section{STUDY DESIGN}

Consent for limited necropsy examination was requested from the parents or guardians of all children aged between 2 months and 15 years dying of respiratory disease in the inpatient wards of UTH. Permission to perform a necropsy limited to the organs of the chest was requested to reduce anxieties about mutilation and to avoid the time delay associated with performing a complete examination. The recruitment officer liaised very closely with the consultant pathologist in charge so that the necropsy was performed within 6 hours of obtaining approval, thus causing minimum delay before the body was released for burial. The examination was limited to the organs of the chest cavity, where the following material was obtained:

- One section from all lobes of the lungs.

- One section from the left and right main bronchus and trachea.

- Hilar lymph nodes.

- Mediastinal lymph nodes.

- One section from the pericardium.

- Cardiac chamber serum sample.

- Oropharyngeal washings.

CONSENT BY PARENT OR GUARDIAN FOR NECROPSY EXAMINATION

Study specific enrolment forms were designed for obtaining necropsy consent. The parents or guardians of all children dying of respiratory disease who were available for interview during daylight working hours in the inpatient wards of the department of paediatrics and child health, UTH Lusaka, were approached by the study recruitment clinical officer and the study paediatrician in charge of the patient. All communication was in the language spoken by the parent or guardian, through our multilingual recruitment officer. Details of the study were explained to the parent/guardian, written information on the study in the appropriate language was given to them, and time to consult any relatives present and come back with any questions was afforded. It was explained that the study would require the deceased child to be HIV tested. Arrangements to offer counselling to parents wishing to know the HIV status of their infant were in place. When the parent/guardian refused permission for necropsy, the main reason given was recorded. There were no predetermined categories of reasons for refusal.

Parents/guardians were encouraged to express their specific concerns about necropsy examination in their own words. For those who consented to a necropsy examination on their child, a lunch was provided for waiting relatives in recognition of the potential stress and inconvenience imposed, and a standard allowance was given to transport the cadaver home.

ETHICS APPROVAL

The study was approved by the research and ethics committee of the University Teaching Hospital and the University of Zambia School of Medicine.

DATA MANAGEMENT AND ANALYSIS

The following information was obtained and maintained on a study register. The parents/ guardians responses were documented by hand as a narrative and later transferred to a word processor, as follows:

- Number of children who died of respiratory diseases in hospital during the study period.

- Number of parents who were approached for consent to perform a necropsy.

- Number of refusals.

- Reasons for refusal. 
Table 1 Reasons given by 891 parents or guardians for refusing permission for a necropsy examination

\begin{tabular}{lll}
\hline Reason & Number & Percentage \\
\hline Waste of time since it will not benefit them & 383 & $43 \%$ \\
Transport already organised and death certificate issued & 236 & $26.5 \%$ \\
Traditional beliefs that ancestral spirits prohibit necropsy & 77 & $8.6 \%$ \\
Can't consent because the child is not their own and must consult other & 54 & $6 \%$ \\
$\quad$ relatives & & \\
Investigations of cause of death should have been done before death of & 38 & $4.3 \%$ \\
$\quad$ child, not after & 31 & $3.4 \%$ \\
Religious (Moslem) belief of limitation on burial time since death & 30 & $3.4 \%$ \\
Not convinced of the rationale of necropsy examination & 14 & $1.6 \%$ \\
Just being used to teach nursing and medical students & 13 & $1.5 \%$ \\
Child too young for necropsy & 6 & $0.7 \%$ \\
Organs from the child will be sold for transplantation & 9 & $1 \%$ \\
Other reasons: & 6 & \\
Already know that child died from HIV/AIDS & 2 & \\
Selling off child & 1 & \\
Child has suffered enough & $\mathbf{8 9 1}$ & \\
Total & & \\
\hline
\end{tabular}

Results

The total number of children admitted to the UTH with respiratory illness who died during the study period was 1603; the parents/ guardians of 1181 children were approached to request consent to perform a necropsy examination. Of these, $891(75.4 \%)$ refused to give permission, while 290 consented (24.6\%). The reasons for refusal of necropsy were many and varied and are given in table 1 .

Almost half the recently bereaved parents or guardians $(43 \%)$ showed anger at the request for necropsy, refusing on the grounds that it would be a "waste of time" for them as the diagnosis should have been made when the child was alive and the result of this study would now be of no benefit to them.

Another major reason for refusal (26.5\%) was that a death certificate had already been issued and arrangements to transport the body and the family had already been made and could not be delayed.

Traditional beliefs that ancestral spirits forbade the mutilation of dead bodies was cited by 77 of the 891 parents/guardians approached $(8.6 \%)$. Their main concern was that ancestral spirits would make all women in their family infertile should consent for necropsy be given.

The fourth most common reason for refusal was that the child was not their own or they must seek permission from other family members who were not available (6\%).

Thirty seven parents/guardians (4.3\%) said that all investigations should have been done before the child's death and that caused them to be suspicious of the study.

Religious beliefs were not a major cause of refusal $(3.4 \%)$, and those who cited them were Muslims who said they had to bury the body within 24 hours of death.

Other anxieties were expressed as follows:

- parents/guardians were not convinced of the rationale for necropsy examination $(3.4 \%)$;

- the necropsy was only meant to teach nursing and medical students $(1.6 \%)$;

- the child was too young for necropsy $(1.5 \%)$;

- organs from the child would be sold for transplantation $(0.7 \%)$;
- six parents refused on the grounds that they were aware that their child had HIV/ AIDS and consequently they knew the cause of death;

- two said that consenting was tantamount to "selling off" the child (in reference to the monies offered for lunch and transport costs of the funeral);

- one parent said that their child had already suffered enough.

Very few guardians gave consent to having a necropsy performed on the child under their care. Younger parents found it more difficult to give consent than older ones, and parents of children younger than 12 months found it easier to give consent than those with older children. It was significant to note that no parents or guardians directly expressed anxieties regarding their own HIV status as the main reason for refusing to consent to necropsy examination of their child. This factor undoubtedly played a part but is not quantifiable from the study findings.

\section{Discussion}

Clinical necropsies cannot be performed without the written permission of the deceased's relatives, and this is the most important enabling step in the necropsy procedure. This study shows that even within a controlled study, where conditions for the rapid performance of a restricted necropsy were optimal, refusal of consent remained common. Over $70 \%$ of the parents or guardians approached refused to allow a necropsy examination on their child. There is little published work on this subject, particularly in children, and no previous studies could be found from Africa assessing the reasons for necropsy refusal. Thus comparisons with other studies are difficult. In Zambia the only available data-from a retrospective study of necropsies at UTH in Lusaka-predate the HIV/AIDS epidemic. ${ }^{15}$ That study found that only $10 \%$ of hospital childhood deaths had necropsies.

The necropsy examination is important to science, to clinical practice, and to bereaved families and carers, ${ }^{16}{ }^{17}$ yet it is uncommonly employed in sub-Saharan Africa owing to the limited availability of clinical pathology services and the social taboos and cultural demands affecting the uptake and development of such a service. Consenting to necropsy examination, particularly for children, is psychologically distressing for all parties involved. Many physicians find the request for necropsy examination to be one of the most difficult and unpleasant aspects of paediatrics. ${ }^{18}$ For those with little or no insight into its scientific rationale the necropsy examination is commonly perceived in terms of disfigurement and mutilation. Challenging perceptions of the procedureparticularly at times of extreme stress, personal tragedy, and grief - may lead to conflict between family members and appears only to exacerbate the stress of bereavement. Confronted by such situations doctors may be reluctant to pursue a dialogue of persuasion that radically challenges the sensibilities of grieving parents, and may elect to avoid or omit requesting permission for necropsy in the future. 
In Zambia traditional beliefs and cultural practices coexist with religious beliefs and modern scientific rationalism. The majority of people use these models in combination when dealing with dying and bereavement. Relatives will seek information about the cause of unnatural death from doctors, traditional healers, and religious leaders. Premature death in particular carries special significance to those whose belief system is strongly influenced by traditional concepts of ancestral spirits, and it can be attributed to witchcraft or punishment for wrongdoing. Age, ethnicity, income, education, and religious beliefs interact to affect attitudes to necropsy examination.

Insight into parents' willingness to consent, as a reflection of local sociocultural diversity, was also important to ensure that our study was representative and excluded no significant group within the population served. Religious objection is often cited as a cause for declining necropsy rates but few religions take a stand against necropsy examinations. ${ }^{19}$ The holy Koran places no restriction against carrying them out, but because of Islamic respect for the dead the body is expected to be buried within 24 hours of death. It thus becomes important to perform the necropsy as a matter of urgency. Within the current constraints on pathology services in most African countries, this is seldom possible.

The skills necessary to make an appropriate request for a necropsy examination and to communicate its value to recently bereaved relatives are complex. Any approach must give full consideration to local beliefs and to social and religious values and customs, and must remain mindful of the procedure's objectives - to verify the cause of death, study the effects of treatment, and sensitively communicate the findings to the bereaved. In Zambia, there are no published guidelines to help families and physicians deal with this difficult area, and no formal instruction about obtaining necropsy consent occurs in medical training. Staff are therefore unprepared for and unsupported in dealing with this most difficult task.

When the study was initially designed, the experience of doctors working at UTH suggested that parents would be extremely reluctant to consent to necropsy examination on their children, and thus the study would be a failure because of non-recruitment. Indeed many doctors do not themselves see the value of necropsy examinations, especially in HIV positive children where the diagnosis of AIDS was obvious. Seeking an aetiology specific diagnosis in a system where resources do not exist to diagnose and treat these conditions has made some doctors and health workers fatalistic and consequently reluctant to request necropsies.

Lay perceptions about the necropsy examination are not well informed in Zambia, and communicating its social and scientific value was challenging. This study benefited from the presence of a mature experienced counsellor who had excellent interpersonal and linguistic

\section{Key messages}

- Worldwide necropsy rates are declining in both adults and children

- Even in conditions where trained counsellors and rapid access to necropsy examination exist, rates of consent remain low

- In Zambia, necropsy examination of children is most commonly perceived by parents/guardians as a waste of time and an inconvenience to arrangements for burial

skills and insight into the complex factors that were likely to influence the parents' decision. Obtaining permission for necropsy examination requires a sympathetic approach by clinicians who themselves must understand the value of the necropsy in clinical problem solving and audit. If the decline in necropsy rates worldwide is to be stemmed, this must begin at medical school where the role and value of the necropsy must be emphasised in the curriculum. Skills in bereavement counselling must be taught at an early stage of medical training in order to improve interpersonal communication between relatives and the treating physician, who will most probably be the person to make the request for necropsy. Good communication has been rightly cited as the most important determinant affecting willingness to consent to necropsy.

This study also raises the question of who is best qualified to counsel the bereaved family and discuss the rationale for necropsy examination with a view to obtaining consent. The study was made possible by the skilful and empathic communication of a multilingual recruitment officer and the efforts of the study team to reduce delay and inconvenience to the family to a minimum. Such resources are not readily available within routine clinical practice in sub-Saharan Africa. Despite the fact that only one quarter of those approached consented to a necropsy examination of their child, this study represents a major achievement and provides a conceptual framework within which to consider issues affecting the uptake of clinical pathology services both locally and further afield.

This study was supported by grant RD443 from the United Kingdom Department for International Development.

1 Laissue J-A, Altermatt HJ, Zurcher B, et al. The significance of the autopsy: evaluation of current autopsy results by the clinician. Schweiz Med Wochenschr 1986;116:130-4.

2 Gobbato F, Vecchiet F, Barbierato D, et al. Inaccuracy of death certificate diagnoses in malignancy: an analysis of 1405 autopsied cases. Hum Pathol 1982;13:1036-8.

3 Anderson RE, Hill RB. The current status of the autopsy in academic medical centers in the United States. Am $\mathcal{F}$ Clin Pathol 1989;92(suppl 1):S31-7.

4 Luglia A, Anabitarte M, Beer JH. Effect of simple interventions on necropsy rate when active informed consent is required. Lancet 1999;354:1391.

5 Pathmanathan R, Chandrasekharan N. Declining postmortems: a cause for concern. Med $\mathcal{f}$ Malaysia 1985;40: 267-70.

6 Solomon SA, Adams KH. Attitudes of relatives to autopsies of elderly patients. Age Ageing 1993;22:205-8.

7 McKelvie PA, Rode J. Autopsy rate and a clinicopathological audit in an Australian metropolitan hospital - cause for concern? Med $\mathcal{f}$ Aust 1992:156:456-62. 
8 Lucas SB, Hounnou A, Peacock C, et al. The mortality and pathology of HIV infection in a west African city. AIDS

9 Lucas SB, Peacock CS, Hounnou A, et al. Disease in children infected with HIV in Abidjan, Cote d'Ivoire. $B M \mathcal{F}$ 1996;312:335-8.

10 Kamali A, Wagner HU, Nakiyingi J, et al. Verbal autopsy as a tool for diagnosing HIV-related adult deaths in rura Uganda. Int $\mathcal{F}$ Epidemiol 1996;25:679-84.

11 Nykanen M, Tamaona W, Cullinan T, et al. Verbal autopsy as a technique to establish causes of infant and child mortality. East Afr Med F 1995;72:731-4.

12 Mobley CC, Boerma JT, Titus S, et al. Validation study of a verbal autopsy method for causes of childhood mortality in Namibia. F Trop Pediatr 1996:42:365-9.

13 Quigley Ma, Armstrong Schellenberg JR, Snow RW. Algorithms for verbal autopsies: a validation study in Kenyan children. Bull WHO 1996;74:147-54.
14 Amakiri CN, Akang EE, Aghadiuno PU, et al. A prospective study of coroner's autopsies in University College Hospital, Ibadan, Nigeria. Med Sci Law 1997;37:1:69-75.

15 Gohill R, Nkanza NK, Bhagwandeen SB, et al. A review of autopsies at the University Teaching Hospital, Lusaka, Zambia. Med f Zambia 1979;13:28-32.

6 Beckwith JB. The value of the pediatric postmortem examination. Pediatr Clin North Am 1989;36:29-36.

17 Cartlidge PH, Dawson AT, Stewart JH, et al. Value and quality of perinatal and infant postmortem examinations: cohort analysis of 400 consecutive deaths. BMF 1995;310: $155-8$.

18 Berger LR. Requesting the autopsy: a paediatric perspective. Psychosocial and professional aspects of the autopsy in caring for the dying child and his family. Clin Pediatr 1978;17: $445-52$.

19 Svendsen E, Hill RB. Autopsy legislation and practice in various countries. Arch Pathol Lab Med 1987;111:846-50.

\section{Thunder, Serenity, and Zen}

It seems inevitable that the marketing of recreational drugs, especially through the Internet, will keep one step ahead of attempts to control them. An illustrative tale centres on $\gamma$-hydroxybutyrate and its precursors (Deborah L Zvosec and colleagues. New England fournal of Medicine 2001;344:87-94).

Gamma-hydroxybutyrate is a metabolite of the inhibitory neurotransmitter, $\gamma$-aminobutyric acid (GABA) and, to some extent, may be reconverted back to GABA. In the 1980s it was promoted as a muscle builder and promoter of fat loss. Because it also induced euphoria and was said to enhance sexual performance it became popular, but toxic effects led to it being banned in the USA although it is said that it is still widely used at "raves". Subsequently, two industrial solvents , 1,4-butanediol and $\gamma$-butyrolactone, both of which are converted to $\gamma$-hydroxybutyrate after ingestion, were marketed as dietary supplements (a 1994 US Act makes it legal to market non-food, non-drug, dietary supplements without proof of safety). Gamma-butyrolactone dietary supplements were subsequently recalled after FDA warnings but 1,4-butanediol was then promoted as a replacement. Further attempts at regulation have led to these compounds being advertised on the Internet as "non-toxic" solvents and cleaners. Proprietary and street names for the compounds include Rejuv@Nite, Liquid Gold, Zen, Thunder, Serenity, Easy Lay, Gamma Ram, Great Hormones at Bedtime, and Liquid Ecstasy. The toxic effects are similar to those of severe alcohol poisoning and include coma, respiratory depression, and death.

Over a period of 6 or 7 months in 1999 nine episodes of acute poisoning with 1,4-butanediol affecting eight patients aged 22-51 were identified through three emergency departments in Minnesota, Texas, and Florida. Symptoms and signs included vomiting, aggressiveness, incontinence of urine and faeces, fluctuating conscious level, and respiratory depression. Two patients died.

The US Drug Enforcement Administration has recorded 71 deaths from $\gamma$-hydroxybutyrate alone or in association with other drugs. Internet sites have tended to minimise the dangers and one apparently advised its readers that the side effects included "short-term, harmless coma".

The writers of this report conclude that 1,4 butanediol "is toxic, addictive, and potentially lethal. Ingestion . . impairs one's ability to drive and it may be used to facilitate sexual assault". Those who use or sell it will no doubt point out that all these things apply to alcohol.

ARCHIVIST 